\title{
A Regularization of the Backward Problem for Nonlinear Parabolic Equation with Time-Dependent Coefficient
}

\author{
Pham Hoang Quan, ${ }^{1}$ Le Minh Triet, ${ }^{1}$ and Dang Duc Trong ${ }^{2}$ \\ ${ }^{1}$ Department of Mathematics and Applications, Sai Gon University, 273 An Duong Vuong, \\ District 5, Ho Chi Minh City, Vietnam \\ ${ }^{2}$ Department of Mathematics, University of Natural Science, Vietnam National University, \\ 227 Nguyen Van Cu, District 5, Ho Chi Minh City, Vietnam
}

Correspondence should be addressed to Le Minh Triet, lmtriet2005@yahoo.com.vn

Received 31 March 2012; Revised 21 June 2012; Accepted 11 August 2012

Academic Editor: Theodore E. Simos

Copyright (c) 2012 Pham Hoang Quan et al. This is an open access article distributed under the Creative Commons Attribution License, which permits unrestricted use, distribution, and reproduction in any medium, provided the original work is properly cited.

We study the backward problem with time-dependent coefficient which is a severely ill-posed problem. We regularize this problem by combining quasi-boundary value method and quasireversibility method and then obtain sharp error estimate between the exact solution and the regularized solution. A numerical experiment is given in order to illustrate our results.

\section{Introduction}

We consider the inverse time problem for the nonlinear parabolic equation

$$
\begin{aligned}
u_{t}(x, t)-a(t) u_{x x}(x, t) & =f(x, t, u(x, t)), \quad(x, t) \in[0, \pi] \times(0, T], \\
u(0, t) & =u(\pi, t)=0, \quad t \in[0, T], \\
u(x, T) & =g(x), \quad x \in[0, \pi]
\end{aligned}
$$

where $a(t)$ is thermal conductivity function of (1.1) such that there exist $p, q>0$ satisfying

$$
0<p \leq a(t) \leq q,
$$

for all $t \in[0, T]$. 
In other words, from the temperature distribution at a particular time $t=T$ (final data), we want to retrieve the temperature distribution at any earlier time $t<T$. This problem is called the backward heat problem (BHP), or final-value problem. As known, this problem is severely ill-posed in Hadamard's sense; that is, solutions do not always exist, and when they exist, they do not depend continuously on the given data. In practice, datum $g$ is based on (physical) measurements. Hence, there will be measurement errors, and we would actually have datum function $g_{\delta}$ such that $\left\|g_{\delta}-g\right\|_{L^{2}(0, \pi)} \leq \delta$. Thus, form small error contaminating physical measurements, the solutions corresponding to datum function $g_{\delta}$ have large errors. This makes it difficult to make numerical calculations with perturbed data.

In our knowledge, there have been many papers on the linear homogeneous case of the backward problem, but there are a few papers on the nonhomogeneous case and the nonlinear case such as [1-6]; especially, the nonlinear case with time-dependent thermal conductivity coefficient is very scarce. Moreover, the thermal conductivity is the property of a material's ability to conduct heat. Therefore, the thermal conductivity is not a constant in some cases. In this paper, we extend the result (in [7]) for the case of the time-dependent thermal conductivity $a(t)$. In future, we will research the BHP problem for the case of the time- and spacedependent thermal conductivity $a(x, t)$.

In [8], the authors used the quasi-reversibility method to regularize a 1D linear nonhomogeneous backward problem. Very recently, in [9], the methods of integral equations and of Fourier transform have been used to solve a 1D problem in an unbounded region. In recent articles considering the nonlinear backward heat problem, we refer the reader to [10]. In [11], the authors used the quasi-boundary value method to regularize the latter problem. However, in [11], the authors showed that the error between the regularized solution and the exact solution is

$$
\left\|u_{\delta}(\cdot, t)-u(\cdot, t)\right\| \leq K(t) \delta^{t / T}, \quad 0<t<T
$$

It is easy to see that the convergence of the error estimate between the regularized solution and the exact solution is very slow when $t$ is in a neighborhood of zero. For this reason, the error estimate in initial time is given by

$$
\left\|u_{\delta}(\cdot, t)-u(\cdot, t)\right\| \leq K\left(\frac{1}{\ln (1 / \delta)}\right)^{1 / 4}
$$

We can easily find that the exact solution of (1.1)-(1.3) satisfies

$$
u(x, t)=\sum_{k=1}^{\infty} u_{k}(t) \sin (k x)
$$

where

$$
\begin{aligned}
u_{k}(t) & =e^{k^{2}(\lambda(T)-\lambda(t))} g_{k}-\int_{t}^{T} e^{k^{2}(\lambda(s)-\lambda(t))} f_{k}(u)(s) d s, \\
f_{k}(u)(s) & =\frac{2}{\pi} \int_{0}^{\pi} f(x, s, u(x, s)) \sin (k x) d x,
\end{aligned}
$$




$$
\begin{aligned}
& g_{k}=\frac{2}{\pi} \int_{0}^{\pi} g(x) \sin (k x) d x, \\
& \lambda(t)=\int_{0}^{t} a(s) d s .
\end{aligned}
$$

In this paper, we will approximate (1.1)-(1.3) by using the regularization problem:

$$
\begin{aligned}
\left(u_{\delta}\right)_{t}(x, t)-a(t)\left(u_{\delta}\right)_{x x}(x, t) & =\sum_{k=1}^{\infty} \frac{e^{-k^{2} \lambda(T)}}{\alpha_{k}(\delta)+e^{-k^{2} \lambda(T)}} f_{k}\left(u_{\delta}\right)(t) \sin (k x), \\
u_{\delta}(0, t) & =u_{\delta}(\pi, t)=0 \\
u_{\delta}(x, T) & =\sum_{k=1}^{\infty} \frac{e^{-k^{2} \lambda(T)}}{\alpha_{k}(\delta)+e^{-k^{2} \lambda(T)}} g_{k} \sin (k x),
\end{aligned}
$$

where $\alpha_{k}(\delta)=\delta k^{2}$. Actually, in [12], we also considered the problem (1.1)-(1.3) for the homogeneous case $f=0$ in $\mathbb{R}$. Hence, we want to extend for the nonlinear case $f(x, t, u)$ in bounded region $[0, \pi]$, and this is the biggest different point in this paper.

The remainder of this paper is organized as follows. In Section 2, we shall regularize the ill-posed problem (1.1)-(1.3) and give the error estimate between the regularized solution and the exact solution. Then, in Section 3, a numerical example is given.

\section{Regularization and Error Estimates}

For clarity of notation, we denote the solution of (1.1)-(1.3) by $u(x, t)$ and the solution of the regularized problem (1.12) by $u_{\delta}(x, t)$. Throughout this paper, we denote $\lambda_{1}=\max \{1, \lambda(T)\}$, and, $\delta$ be a positive number such that $0<\delta<\lambda(T)$. Hereafter, we have a number of inequalities in order to evaluate error estimates.

Lemma 2.1. Let $a(t)$ be a function satisfying $(1.4), \alpha_{k}(\delta)=\delta k^{2}, B_{\delta}=(\delta \ln (\lambda(T) / \delta))^{-1}$ and $\lambda(t)$ be as in (1.11). Then for $k>0$ and $0 \leq t \leq s \leq T$, one gets

$$
\begin{aligned}
& \text { (i) } e^{k^{2}(\lambda(s)-\lambda(t)-\lambda(T))} /\left(\alpha_{k}(\delta)+e^{-k^{2} \lambda(T)}\right) \leq \lambda_{1}(\delta \ln (\lambda(T) / \delta))^{(\lambda(t)-\lambda(s)) / \lambda(T)}= \\
& \quad \lambda_{1}\left(B_{\delta}\right)^{(\lambda(s)-\lambda(t)) / \lambda(T)}, \\
& \text { (ii) } e^{-k^{2} \lambda(t)} /\left(\alpha_{k}(\delta)+e^{-k^{2} \lambda(T)}\right) \leq \lambda_{1}(\delta \ln (\lambda(T) / \delta))^{(\lambda(t)-\lambda(T)) / \lambda(T)}=\lambda_{1}\left(B_{\delta}\right)^{(\lambda(T)-\lambda(t)) / \lambda(T)} .
\end{aligned}
$$

Proof of Lemma 2.1. The proof of Lemma 2.1 can be found in [12].

Theorem 2.2. Let $g \in L^{2}(0, \pi)$ and $f \in L^{\infty}([0, \pi] \times[0, T] \times R)$ such that there exists $L>0$ independent of $x, t, u, v$ satisfying

$$
|f(x, t, u)-f(x, t, v)| \leq L|u-v|
$$


Then problem (1.12) has a unique weak solution $u_{\delta} \in W=C\left([0, T] ; L^{2}(0, \pi)\right)$ satisfying the following equality:

$$
u_{\delta}(x, t)=\sum_{k=1}^{\infty}\left[\frac{e^{-k^{2} \lambda(t)}}{\alpha_{k}(\delta)+e^{-k^{2} \lambda(T)}} g_{k}-\int_{t}^{T} \frac{e^{k^{2}(\lambda(s)-\lambda(t)-\lambda(T))}}{\alpha_{k}(\delta)+e^{-k^{2} \lambda(T)}} f_{k}\left(u_{\delta}\right)(s) d s\right] \sin (k x),
$$

where

$$
\begin{gathered}
g_{k}=\frac{2}{\pi} \int_{0}^{\pi} g(x) \sin (k x) d x \\
f_{k}\left(u_{\delta}\right)(s)=\frac{2}{\pi} \int_{0}^{\pi} f\left(x, s, u_{\delta}\right) \sin (k x) d x .
\end{gathered}
$$

Proof of Theorem 2.2. Step 1. The existence and the uniqueness of the solution of the problem (2.2).

Put

$$
F(u)(x, t)=\sum_{k=1}^{\infty}\left(P_{k}(t)-K_{k}(u)(t)\right) \sin (k x),
$$

where

$$
\begin{gathered}
P_{k}(t)=\frac{e^{-k^{2} \lambda(t)}}{\alpha_{k}(\delta)+e^{-k^{2} \lambda(T)}} g_{k}, \\
K_{k}(u)(t)=\int_{t}^{T} \frac{e^{k^{2}(\lambda(s)-\lambda(t)-\lambda(T))}}{\alpha_{k}(\delta)+e^{-k^{2} \lambda(T)}} f_{k}(u)(s) d s .
\end{gathered}
$$

We claim that, for every $u, v \in C\left([0, T] ; L^{2}(0, \pi)\right), k \geq 1$, we have

$$
\left\|F^{k}(u)(\cdot, t)-F^{k}(v)(\cdot, t)\right\|^{2} \leq \frac{(T-t)^{k}}{k !}\left(B_{\delta}\right)^{2 k q / p} C \lambda_{1}^{2 k} L^{2 k}\|u-v\|_{C\left([0, T] ; L^{2}(0, \pi)\right)^{\prime}}^{2}
$$

where $C=\max \{1, T\}$ and $\|\cdot\|_{C\left([0, T] ; L^{2}(0, \pi)\right)}$ is supremum norm in $C\left([0, T] ; L^{2}(0, \pi)\right)$. We shall prove this inequality by induction. For $k=1$, we have

$$
\begin{aligned}
& \|F(u)(\cdot, t)-F(v)(\cdot, t)\|^{2} \\
& =\frac{\pi}{2} \sum_{k=1}^{\infty}\left(K_{k}(u)(t)-K_{k}(v)(t)\right)^{2}
\end{aligned}
$$




$$
\begin{aligned}
& =\frac{\pi}{2} \sum_{k=1}^{\infty}\left[\int_{t}^{T} \frac{e^{k^{2}(\lambda(s)-\lambda(t)-\lambda(T))}}{\alpha_{k}(\delta)+e^{-k^{2} \lambda(T)}}\left(f_{k}(u)(s)-f_{k}(v)(s)\right) d s\right]^{2} \\
& \leq \frac{\pi}{2} \sum_{k=1}^{\infty}\left[\int_{t}^{T}\left(\frac{e^{k^{2}(\lambda(s)-\lambda(t)-\lambda(T))}}{\alpha_{k}(\delta)+e^{-k^{2} \lambda(T)}}\right)^{2} d s \int_{t}^{T}\left(f_{k}(u)(s)-f_{k}(v)(s)\right)^{2} d s\right] .
\end{aligned}
$$

From Lemma 2.1, we get

$$
\begin{aligned}
& \|F(u)(\cdot, t)-F(v)(\cdot, t)\|^{2} \\
& \quad \leq \frac{\pi}{2} \sum_{k=1}^{\infty}\left[\int_{t}^{T}\left(\lambda_{1}\left(\delta \ln \left(\frac{\lambda(T)}{\delta}\right)\right)^{(\lambda(t)-\lambda(s)) / \lambda(T)}\right)^{2} d s \int_{t}^{T}\left(f_{k}(u)(s)-f_{k}(v)(s)\right)^{2} d s\right] .
\end{aligned}
$$

It follows

$$
\begin{aligned}
\|F(u)(\cdot, t)-F(v)(\cdot, t)\|^{2} \\
\leq \frac{\pi}{2} \sum_{k=1}^{\infty}\left[\int_{t}^{T} \lambda_{1}^{2}\left(B_{\delta}\right)^{2(\lambda(s)-\lambda(t)) / \lambda(T)} d s \int_{t}^{T}\left(f_{k}(u)(s)-f_{k}(v)(s)\right)^{2} d s\right] \\
\leq \frac{\pi}{2} \sum_{k=1}^{\infty}\left[\int_{t}^{T} \lambda_{1}^{2}\left(B_{\delta}\right)^{2(q s-p t) / p T} d s \int_{t}^{T}\left(f_{k}(u)(s)-f_{k}(v)(s)\right)^{2} d s\right] \\
\leq \frac{\pi}{2} \sum_{k=1}^{\infty}\left[\int_{t}^{T} \lambda_{1}^{2}\left(B_{\delta}\right)^{2 q / p} d s \int_{t}^{T}\left(f_{k}(u)(s)-f_{k}(v)(s)\right)^{2} d s\right] \\
\quad=\lambda_{1}^{2} B_{\delta}^{2 q / p}(T-t) \int_{t}^{T} \int_{0}^{\pi}(f(x, s, u(x, s))-f(x, s, v(x, s)))^{2} d x d s .
\end{aligned}
$$

Therefore, we have

$$
\begin{aligned}
& \|F(u)(\cdot, t)-F(v)(\cdot, t)\|^{2} \\
& \quad \leq \lambda_{1}^{2} L^{2} B_{\delta}^{2 q / p}(T-t) \int_{t}^{T} \int_{0}^{\pi}(u(x, s)-v(x, s))^{2} d x d s \\
& \quad \leq(T-t) B_{\delta}^{2 q / p} C \lambda_{1}^{2} L^{2}\|u-v\|_{C\left([0, T] ; L^{2}(0, \pi)\right)^{\prime}}^{2}
\end{aligned}
$$

where $C=\max \{1, T\}$. 
Thus, (2.6) holds for $k=1$. Supposing that (2.6) holds for $k=n$, we shall prove that (2.6) holds for $k=n+1$. In fact, we get

$$
\begin{aligned}
\left\|F^{n+1}(u)(\cdot, t)-F^{n+1}(v)(\cdot, t)\right\|^{2} \\
\quad=\frac{\pi}{2} \sum_{k=1}^{\infty}\left[K_{k}\left(F^{n}(u)\right)(t)-K_{k}\left(F^{n}(v)\right)(t)\right]^{2} \\
=\frac{\pi}{2} \sum_{k=1}^{\infty}\left(\int_{t}^{T} \frac{e^{k^{2}(\lambda(s)-\lambda(t)-\lambda(T))}}{\alpha_{k}(\delta)+e^{-k^{2} \lambda(T)}}\left(f_{k}\left(F^{n}(u)\right)(s)-f_{k}\left(F^{n}(v)\right)(s)\right) d s\right)^{2} .
\end{aligned}
$$

Hence, we obtain

$$
\begin{aligned}
& \left\|F^{n+1}(u)(x, t)-F^{n+1}(v)(x, t)\right\|^{2} \\
& \quad \leq \frac{\pi}{2}\left(B_{\delta}\right)^{2 q / p} \lambda_{1}^{2}(T-t) \int_{t}^{T} \sum_{k=1}^{\infty}\left(f_{k}\left(F^{n}(u)\right)(s)-f_{k}\left(F^{n}(v)\right)(s)\right)^{2} d s \\
& \quad=\left(B_{\delta}\right)^{2 q / p} \lambda_{1}^{2}(T-t) \int_{t}^{T}\left\|f\left(\cdot, s, F^{n}(u)(\cdot, s)\right)-f_{k}\left(\cdot, s, F^{n}(v)(\cdot, s)\right)\right\|^{2} d s \\
& \quad \leq\left(B_{\delta}\right)^{2 q / p} \lambda_{1}^{2}(T-t) L^{2} \int_{t}^{T}\left\|F^{n}(u)(\cdot, s)-F^{n}(v)(\cdot, s)\right\|^{2} d s .
\end{aligned}
$$

Thus, we have

$$
\begin{aligned}
\left\|F^{n+1}(u)(x, t)-F^{n+1}(v)(x, t)\right\|^{2} \\
\quad \leq\left(B_{\delta}\right)^{2 q / p} \lambda_{1}^{2}(T-t) L^{2} \int_{t}^{T} L^{2 n}\left(B_{\delta}\right)^{2 n q / p} \lambda_{1}^{2 n} \frac{(T-s)^{n}}{n !} C^{n}\|u-v\|_{C\left([0, T] ; L^{2}(0, \pi)\right)}^{2} d s \\
\quad \leq C^{n}\left(B_{\delta}\right)^{2(n+1) q / p} \lambda_{1}^{2 n+2}(T-t) L^{2 n+2}\|u-v\|_{C\left([0, T] ; L^{2}(0, \pi)\right)}^{2} \int_{t}^{T} \frac{(T-s)^{n}}{n !} d s \\
\quad \leq \frac{(T-t)^{n+1}}{(n+1) !} C^{n+1}\left(B_{\delta}\right)^{2(n+1) q / p} \lambda_{1}^{2 n+2}(T-t) L^{2 n+2}\|u-v\|_{C\left([0, T] ; L^{2}(0, \pi)\right)}^{2} .
\end{aligned}
$$

Therefore, by the induction principle, we have

$$
\left\|F^{k}(u)(x, t)-F^{k}(v)(x, t)\right\| \leq \frac{(T-t)^{k / 2}}{\sqrt{k !}}\left(B_{\delta}\right)^{k q / p} C^{k / 2} \lambda_{1}^{k} L^{k}\|u-v\|_{C\left([0, T] ; L^{2}(0, \pi)\right)}
$$

for all $u, v \in C\left([0, T] ; L^{2}(0, \pi)\right)$. 
International Journal of Mathematics and Mathematical Sciences

We consider $F: C\left([0, T] ; L^{2}(0, \pi)\right) \rightarrow C\left([0, T] ; L^{2}(0, \pi)\right)$. Since

$$
\frac{(T-t)^{k / 2}}{\sqrt{k !}}\left(B_{\delta}\right)^{k q / p} \lambda_{1}^{k} C^{k / 2} L^{k} \longrightarrow 0
$$

when $k \rightarrow \infty$, there exists a positive integer number $k_{0}$ such that

$$
\frac{(T-t)^{k_{0} / 2}}{\sqrt{k_{0} !}}\left(B_{\delta}\right)^{k_{0} q / p} \lambda_{1}^{k_{0}} C^{k_{0} / 2} L^{k_{0}}<1,
$$

and $F^{k_{0}}$ is a contraction. It follows that the equation $F^{k_{0}}(u)=u$ has a unique solution $u_{\delta} \in$ $C\left([0, T] ; L^{2}(0, \pi)\right)$.We claim that $F\left(u_{\delta}\right)=u_{\delta}$. In fact, one has $F\left(F^{k_{0}}\left(u_{\delta}\right)\right)=F\left(u_{\delta}\right)$. Hence, $F^{k_{0}}\left(F\left(u_{\delta}\right)\right)=F\left(u_{\delta}\right)$. By the uniqueness of the fixed point of $F^{k_{0}}$, one has $F\left(u_{\delta}\right)=u_{\delta}$; that is, the equation $F(u)=u$ has a unique solution $u_{\delta} \in C\left([0, T] ; L^{2}(0, \pi)\right)$.

Step 2. If $u_{\delta} \in W$ satisfies (2.2), then $u_{\delta}$ is the solution of the problem (1.12). For $0 \leq t \leq T$, we have

$$
u_{\delta}(x, t)=\sum_{k=1}^{\infty}\left[\frac{e^{-k^{2} \lambda(t)}}{\alpha_{k}(\delta)+e^{-k^{2} \lambda(T)}} g_{k}-\int_{t}^{T} \frac{e^{k^{2}(\lambda(s)-\lambda(t)-\lambda(T))}}{\alpha_{k}(\delta)+e^{-k^{2} \lambda(T)}} f_{k}\left(u_{\delta}\right)(s) d s\right] \sin (k x) .
$$

We can verify directly that $u_{\delta} \in C\left([0, T] ; L^{2}(0, \pi) \cap L^{2}\left(0, T ; H_{0}^{1}(0, \pi)\right) \cap\right.$ $C^{1}\left(0, T ; H_{0}^{1}(0, \pi)\right)$. In fact, $u_{\delta} \in C^{\infty}\left([0, T] ; H_{0}^{1}(0, \pi)\right)$. Moreover, one has

$$
\begin{aligned}
\left(u_{\delta}\right)_{t}(x, t)= & \sum_{k=1}^{\infty}-k^{2} a(t) \frac{\left(e^{-k^{2} \lambda(t)} g_{k}-\int_{t}^{T} e^{k^{2}(\lambda(s)-\lambda(t)-\lambda(T))} f_{k}\left(u_{\delta}\right)(s) d s\right)}{\alpha_{k}(\delta)+e^{-k^{2} \lambda(T)}} \sin (k x) \\
& +\sum_{k=1}^{\infty} \frac{e^{-k^{2} \lambda(T)}}{\alpha_{k}(\delta)+e^{-k^{2} \lambda(T)}} f_{k}\left(u_{\delta}\right)(t) \sin (k x) \\
= & -a(t)\left(u_{\delta}\right)_{x x}(x, t)+\sum_{k=1}^{\infty} \frac{e^{-k^{2} \lambda(T)}}{\alpha_{k}(\delta)+e^{-k^{2} \lambda(T)}} f_{k}\left(u_{\delta}\right)(t) \sin (k x), \\
u_{\delta}(x, T)= & \sum_{k=1}^{\infty} \frac{e^{-k^{2} \lambda(T)}}{\alpha_{k}(\delta)+e^{-k^{2} \lambda(T)}} g_{k} \sin (k x) .
\end{aligned}
$$

Hence, $u_{\delta}$ is the solution of (1.12).

Step 3. The problem (1.12) has at most one solution $u_{\delta} \in C\left([0, T] ; L^{2}(0, \pi) \cap\right.$ $L^{2}\left(0, T ; H_{0}^{1}(0, \pi)\right) \cap C^{1}\left(0, T ; H_{0}^{1}(0, \pi)\right)$. In fact, let $u_{\delta}$ and $v_{\delta}$ be two solutions of (1.12) such that $u_{\delta}, v_{\delta} \in W$. Putting $w_{\delta}(x, t)=u_{\delta}(x, t)-v_{\delta}(x, t)$, then $w^{\delta}$ satisfies

$$
\left(w_{\delta}\right)_{t}-\left(w_{\delta}\right)_{x x}=\sum_{k=1}^{\infty} \frac{e^{-k^{2} \lambda(T)}}{\alpha_{k}(\delta)+e^{-k^{2} \lambda(T)}}\left(f_{k}\left(u_{\delta}\right)(t)-f_{k}\left(v_{\delta}\right)(t)\right) \sin (k x) .
$$


It follows that

$$
\begin{aligned}
\left\|\left(w_{\delta}\right)_{t}-\left(w_{\delta}\right)_{x x}\right\|^{2} & \leq \frac{1}{\delta^{2}} \sum_{k=1}^{\infty}\left(f_{k}\left(u_{\delta}\right)(t)-f_{k}\left(v_{\delta}\right)(t)\right)^{2} \\
& \leq \frac{1}{\delta^{2}}\left\|f\left(\cdot, t, u_{\delta}(\cdot, t)\right)-f\left(\cdot, t, v_{\delta}(\cdot, t)\right)\right\|^{2} \\
& \leq \frac{L^{2}}{\delta^{2}}\left\|u_{\delta}(\cdot, t)-v_{\delta}(\cdot, t)\right\|^{2} \\
& =\frac{L^{2}}{\delta^{2}}\left\|w_{\delta}(\cdot, t)\right\|^{2} .
\end{aligned}
$$

By using the result in Lees and Protter [13], we get $w_{\delta}(\cdot, t)=0$. This completes the proof of Step 3.

Finally, by combining three steps, we complete the proof of Theorem 2.2.

Theorem 2.3 (stability of the modified method). Let fbe as in Theorem $2.2, \alpha_{k}(\delta)=\delta k^{2}$, g and let $g_{\delta}$ in $L^{2}(0, \pi)$ satisfy $\left\|g_{\delta}-g\right\| \leq \delta$. If one supposes that $u_{\delta}$ and $v_{\delta}$ defined by $(2.2)$ are corresponding to the final values $g$ and $g_{\delta}$ in $L^{2}(0, \pi)$, respectively, then one obtains

$$
\left\|u_{\delta}(\cdot, t)-v_{\delta}(\cdot, t)\right\| \leq \sqrt{2} \lambda_{1} e^{L^{2} \lambda_{1}^{2} T(T-t)} \delta^{\lambda(t) / \lambda(T)}\left(\ln \left(\frac{\lambda(T)}{\delta}\right)\right)^{(\lambda(t)-\lambda(T)) / \lambda(T)} .
$$

Proof of Theorem 2.3. Using the inequality $(a+b)^{2} \leq 2\left(a^{2}+b^{2}\right)$ and Lemma 2.1, we get the estimate

$$
\begin{aligned}
\| u_{\delta}(\cdot, t) & -v_{\delta}(\cdot, t) \|^{2} \\
\leq & \pi \sum_{k=1}^{\infty}\left|\frac{e^{-k^{2} \lambda(t)}}{\alpha_{k}(\delta)+e^{-k^{2} \lambda(T)}}\left(g_{k}-g_{k, \delta}\right)\right|^{2} \\
& +\pi \sum_{k=1}^{\infty}\left|\int_{t}^{T} \frac{e^{k^{2}(\lambda(s)-\lambda(t)-\lambda(T))}}{\alpha_{k}(\delta)+e^{-k^{2} \lambda(T)}}\left(f_{k}\left(u_{\delta}\right)(s)-f_{k}\left(v_{\delta}\right)(s)\right) d s\right|^{2} \\
\leq & \pi \sum_{k=1}^{\infty}\left|\frac{e^{-k^{2} \lambda(t)}}{\alpha_{k}(\delta)+e^{-k^{2} \lambda(T)}}\left(g_{k}-g_{k, \delta}\right)\right|^{2} \\
& +\pi(T-t) \sum_{k=1}^{\infty} \int_{t}^{T}\left(\frac{e^{k^{2}(\lambda(s)-\lambda(t)-\lambda(T))}}{\alpha_{k}(\delta)+e^{-k^{2} \lambda(T)}}\left(f_{k}\left(u_{\delta}\right)(s)-f_{k}\left(v_{\delta}\right)(s)\right)\right)^{2} d s .
\end{aligned}
$$


Thus, we get

$$
\begin{aligned}
\| u_{\delta}(\cdot, t) & -v_{\delta}(\cdot, t) \|^{2} \\
\leq & \pi \lambda_{1}^{2}\left(B_{\delta}\right)^{2(\lambda(T)-\lambda(t)) / \lambda(T)} \sum_{k=1}^{\infty}\left|g_{k}-g_{k, \delta}\right|^{2} \\
& +\pi(T-t) \sum_{k=1}^{\infty} \int_{t}^{T} \lambda_{1}^{2}\left(B_{\delta}\right)^{2(\lambda(s)-\lambda(t)) / \lambda(T)}\left|f_{k}\left(u_{\delta}\right)(s)-f_{k}\left(v_{\delta}\right)(s)\right|^{2} d s .
\end{aligned}
$$

Hence, we obtain

$$
\begin{aligned}
\| u_{\delta}(\cdot, t) & -v_{\delta}(\cdot, t) \|^{2} \\
\leq & \pi \lambda_{1}^{2}\left(B_{\delta}\right)^{2(\lambda(T)-\lambda(t)) / \lambda(T)} \sum_{k=1}^{\infty}\left|g_{k}-g_{k, \delta}\right|^{2} \\
& +\lambda_{1}^{2} \pi T\left(B_{\delta}\right)^{2(\lambda(T)-\lambda(t)) / \lambda(T)} \sum_{k=1}^{\infty} \int_{t}^{T}\left(B_{\delta}\right)^{2(\lambda(s)-\lambda(T)) / \lambda(T)}\left|f_{k}\left(u_{\delta}\right)(s)-f_{k}\left(v_{\delta}\right)(s)\right|^{2} d s \\
\leq & \pi \lambda_{1}^{2}\left(B_{\delta}\right)^{2(\lambda(T)-\lambda(t)) / \lambda(T)} \sum_{k=1}^{\infty}\left|g_{k}-g_{k, \delta}\right|^{2} \\
& +\lambda_{1}^{2} \pi T\left(B_{\delta}\right)^{2(\lambda(T)-\lambda(t)) / \lambda(T)} \int_{t}^{T}\left(B_{\delta}\right)^{2(\lambda(s)-\lambda(T)) / \lambda(T)} \sum_{k=1}^{\infty}\left|f_{k}\left(u_{\delta}\right)(s)-f_{k}\left(v_{\delta}\right)(s)\right|^{2} d s .
\end{aligned}
$$

Thus, we get

$$
\begin{aligned}
&\left(B_{\delta}\right)^{2(\lambda(t)-\lambda(T)) / \lambda(T)}\left\|u_{\delta}(\cdot, t)-v_{\delta}(\cdot, t)\right\|^{2} \\
& \leq 2 \lambda_{1}^{2}\left\|g-g_{\delta}\right\|^{2} \\
&+2 \lambda_{1}^{2} T \int_{t}^{T}\left(B_{\delta}\right)^{2(\lambda(s)-\lambda(T)) / \lambda(T)}\left\|f\left(\cdot, s, u_{\delta}(\cdot, s)\right)-f\left(\cdot, s, v_{\delta}(\cdot, s)\right)\right\|^{2} \\
& \leq 2 \lambda_{1}^{2}\left\|g-g_{\delta}\right\|^{2} \\
&+2 L^{2} \lambda_{1}^{2} T \int_{t}^{T}\left(B_{\delta}\right)^{2(\lambda(s)-\lambda(T)) / \lambda(T)}\left\|u_{\delta}(\cdot, s)-v_{\delta}(\cdot, s)\right\|^{2} .
\end{aligned}
$$

By using Gronwall's inequality, we have

$$
\left(B_{\delta}\right)^{2(\lambda(t)-\lambda(T)) / \lambda(T)}\left\|u_{\delta}(\cdot, t)-v_{\delta}(\cdot, t)\right\|^{2} \leq 2 \lambda_{1}^{2} e^{2 L^{2} \lambda_{1}^{2} T(T-t)}\left\|g_{\delta}-g\right\|^{2} .
$$


It follows

$$
\begin{aligned}
\left\|u_{\delta}(\cdot, t)-v_{\delta}(\cdot, t)\right\| & \leq \sqrt{2} \lambda_{1} e^{L^{2} \lambda_{1}^{2} T(T-t)}\left(B_{\delta}\right)^{(\lambda(T)-\lambda(t)) / \lambda(T)}\left\|g_{\delta}-g\right\| \\
& \leq \sqrt{2} \lambda_{1} e^{L^{2} \lambda_{1}^{2} T(T-t)} \delta^{\lambda(t) / \lambda(T)}\left(\ln \left(\frac{\lambda(T)}{\delta}\right)\right)^{(\lambda(t)-\lambda(T)) / \lambda(T)}
\end{aligned}
$$

This completes the proof of Theorem 2.3.

Theorem 2.4. Let $u$ be the exact solution of problem (1.1)-(1.3) such that

$$
\begin{gathered}
Q(t)=3 \lambda_{1}^{2} e^{3 L^{2} T \lambda_{1}^{2}(T-t)}\left\|u_{x x}(\cdot, 0)\right\|^{2}<\infty, \\
M(t)=6 T \lambda_{1}^{2} e^{3 L^{2} T \lambda_{1}^{2}(T-t)} \int_{0}^{T} \int_{0}^{\pi} \sum_{k=1}^{\infty}\left|k^{2} e^{k^{2} \lambda(s)}\left(u_{s}(x, s)-a(s) u_{x x}(x, s)\right)\right|^{2} d x d s<\infty
\end{gathered}
$$

for all $t \in[0, T)$. Letting $\alpha_{k}(\delta)=\delta k^{2}$ and $v_{\delta}(\cdot, t)$ given by (2.2) corresponding to the perturbed data $g_{\delta}$, then one has for every $t \in[0, T)$

$$
\left\|u(\cdot, t)-v_{\delta}(\cdot, t)\right\| \leq C(t) \delta^{\lambda(t) / \lambda(T)}\left(\ln \left(\frac{\lambda(T)}{\delta}\right)\right)^{(\lambda(t)-\lambda(T)) / \lambda(T)}
$$

where $C(t)=\sqrt{2} \lambda_{1} e^{L^{2} \lambda_{1}^{2} T(T-t)}+\sqrt{Q(t)+M(t)}$.

Proof of Theorem 2.4. From (1.1), we construct the regularized solution corresponding to the exact data and the perturbed data

$$
\begin{aligned}
& u_{\delta}(x, t)=\sum_{k=1}^{\infty} u_{k, \delta}(t) \sin (k x), \\
& v_{\delta}(x, t)=\sum_{k=1}^{\infty} v_{k, \delta}(t) \sin (k x),
\end{aligned}
$$

where

$$
\begin{gathered}
u_{k, \delta}(t)=\frac{e^{-k^{2} \lambda(t)}}{\alpha_{k}(\delta)+e^{-k^{2} \lambda(T)}} g_{k}-\int_{t}^{T} \frac{e^{k^{2}(\lambda(s)-\lambda(t)-\lambda(T))}}{\alpha_{k}(\delta)+e^{-k^{2} \lambda(T)}} f_{k}\left(u_{\delta}\right)(s) d s, \\
v_{k, \delta}(t)=\frac{e^{-k^{2} \lambda(t)}}{\alpha_{k}(\delta)+e^{-k^{2} \lambda(T)}} g_{k, \delta}-\int_{t}^{T} \frac{e^{k^{2}(\lambda(s)-\lambda(t)-\lambda(T))}}{\alpha_{k}(\delta)+e^{-k^{2} \lambda(T)}} f_{k}\left(v_{\delta}\right)(s) d s .
\end{gathered}
$$


Since (1.8) and (2.32), we get

$$
\begin{aligned}
& \left|u_{k}(t)-u_{k, \delta}(t)\right| \\
& =\left|\begin{array}{c}
\frac{\alpha_{k}(\delta) e^{-k^{2} \lambda(t)}}{\alpha_{k}(\delta)+e^{-k^{2} \lambda(T)}} e^{k^{2} \lambda(T)} g_{k}-\int_{t}^{T} \frac{\alpha_{k}(\delta) e^{k^{2}(\lambda(s)-\lambda(t)-\lambda(T))}}{\left(\alpha_{k}(\delta)+e^{-k^{2} \lambda(T)}\right)} e^{k^{2} \lambda(T)} f_{k}(u)(s) d s \\
+\int_{t}^{T} \frac{e^{k^{2}(\lambda(s)-\lambda(t)-\lambda(T))}}{\alpha_{k}(\delta)+e^{-k^{2} \lambda(T)}}\left(f_{k}\left(u_{\delta}\right)(s)-f_{k}(u)(s)\right) d s
\end{array}\right| .
\end{aligned}
$$

From $\alpha_{k}(\delta)=\delta k^{2}$, we get

$$
\begin{aligned}
\mid u_{k}(t)- & u_{k, \delta}(t) \mid \\
\leq & \left|\frac{\delta e^{-k^{2} \lambda(t)}}{\delta k^{2}+e^{-k^{2} \lambda(T)}} k^{2} e^{k^{2} \lambda(T)} g_{k}-\int_{t}^{T} \frac{\delta e^{k^{2}(\lambda(s)-\lambda(t)-\lambda(T))}}{\delta k^{2}+e^{-k^{2} \lambda(T)}} k^{2} e^{k^{2} \lambda(T)} f_{k}(u)(s) d s\right| \\
& +\left|\int_{t}^{T} \frac{e^{k^{2}(\lambda(s)-\lambda(t)-\lambda(T))}}{\delta k^{2}+e^{-k^{2} \lambda(T)}}\left(f_{k}\left(u_{\delta}\right)(s)-f_{k}(u)(s)\right) d s\right| \\
\leq & \left|\frac{\delta e^{-k^{2} \lambda(t)}}{\delta k^{2}+e^{-k^{2} \lambda(T)}}\right|\left|k^{2} e^{k^{2} \lambda(T)} g_{k}-k^{2} \int_{0}^{T} e^{k^{2} \lambda(s)} f_{k}(u)(s) d s\right| \\
& +\left|\frac{\delta e^{-k^{2} \lambda(t)}}{\delta k^{2}+e^{-k^{2} \lambda(T)}}\right| \int_{0}^{T}\left|k^{2} e^{k^{2} \lambda(s)} f_{k}(u)(s)\right| d s \\
& +\left|\int_{t}^{T} \frac{e^{k^{2}(\lambda(s)-\lambda(t)-\lambda(T))}}{\delta k^{2}+e^{-k^{2} \lambda(T)}}\left(f_{k}\left(u_{\delta}\right)(s)-f_{k}(u)(s)\right) d s\right| .
\end{aligned}
$$

By applying the inequality $(a+b+c)^{2} \leq 3\left(a^{2}+b^{2}+c^{2}\right)$, we get

$$
\begin{aligned}
\| u(\cdot, t) & -u_{\delta}(\cdot, t) \|^{2} \\
= & \frac{\pi}{2} \sum_{k=1}^{\infty}\left|u_{k, \delta}(t)-u_{k}(t)\right|^{2} \\
\leq & \frac{3 \pi}{2} \sum_{k=1}^{\infty}\left|\frac{\delta e^{-k^{2} \lambda(t)}}{\delta k^{2}+e^{-k^{2} \lambda(T)}}\right|^{2}\left|k^{2} e^{k^{2} \lambda(T)} g_{k}-k^{2} \int_{0}^{T} e^{k^{2} \lambda(s)} f_{k}(u)(s) d s\right|^{2} \\
& +\frac{3 \pi}{2} \sum_{k=1}^{\infty}\left|\frac{\delta e^{-k^{2} \lambda(t)}}{\delta k^{2}+e^{-k^{2} \lambda(T)}}\right|^{2}\left(\int_{0}^{T}\left|k^{2} e^{k^{2} \lambda(s)} f_{k}(u)(s)\right| d s\right)^{2} \\
& +\frac{3 \pi}{2} \sum_{k=1}^{\infty}\left|\int_{t}^{T} \frac{e^{k^{2}(\lambda(s)-\lambda(t)-\lambda(T))}}{\delta k^{2}+e^{-k^{2} \lambda(T)}}\left(f_{k}\left(u_{\delta}\right)(s)-f_{k}(u)(s)\right) d s\right|^{2} .
\end{aligned}
$$


Using Lemma 2.1, we obtain

$$
\begin{aligned}
\| u(\cdot, t) & -u_{\delta}(\cdot, t) \|^{2} \\
\leq & \frac{3 \pi}{2} \lambda_{1}^{2}\left(L_{1}(t)+L_{2}(t)+L_{3}(t)\right), \\
= & \frac{3 \pi}{2} \lambda_{1}^{2} \delta^{2 \lambda(t) / \lambda(T)}\left(\ln \left(\frac{\lambda(T)}{\delta}\right)\right)^{2(\lambda(t)-\lambda(T)) / \lambda(T)} \sum_{k=1}^{\infty}\left|k^{2} u_{k}(0)\right|^{2} \\
& +\frac{3 \pi}{2} \lambda_{1}^{2} \delta^{2 \lambda(t) / \lambda(T)}\left(\ln \left(\frac{\lambda(T)}{\delta}\right)\right)^{2(\lambda(t)-\lambda(T)) / \lambda(T)} \sum_{k=1}^{\infty}\left(\int_{0}^{T}\left|k^{2} e^{k^{2} \lambda(s)} f_{k}(u)(s)\right| d s\right)^{2} \\
& +\frac{3 \pi}{2} \lambda_{1}^{2} T \delta^{2 \lambda(t) / \lambda(T)}\left(\ln \left(\frac{\lambda(T)}{\delta}\right)\right)^{2(\lambda(t)-\lambda(T)) / \lambda(T)} \\
& \times \int_{t}^{T} \delta^{-2 \lambda(s) / \lambda(T)}\left(\ln \left(\frac{\lambda(T)}{\delta}\right)\right)^{2(\lambda(T)-\lambda(s)) / \lambda(T)} \sum_{k=1}^{\infty}\left|\left(f_{k}\left(u_{\delta}\right)(s)-f_{k}(u)(s)\right)\right|^{2} d s,
\end{aligned}
$$

where

$$
\begin{aligned}
L_{1}(t)= & \delta^{2 \lambda(t) / \lambda(T)}\left(\ln \left(\frac{\lambda(T)}{\delta}\right)\right)^{2(\lambda(t)-\lambda(T)) / \lambda(T)} \sum_{k=1}^{\infty}\left|k^{2} u_{k}(0)\right|^{2}, \\
L_{2}(t)= & \delta^{2 \lambda(t) / \lambda(T)}\left(\ln \left(\frac{\lambda(T)}{\delta}\right)\right)^{2(\lambda(t)-\lambda(T)) / \lambda(T)} \sum_{k=1}^{\infty}\left(\int_{0}^{T}\left|k^{2} e^{k^{2} \lambda(s)} f_{k}(u)(s)\right| d s\right)^{2}, \\
L_{3}(t)= & T \delta^{2 \lambda(t) / \lambda(T)}\left(\ln \left(\frac{\lambda(T)}{\delta}\right)\right)^{2(\lambda(t)-\lambda(T)) / \lambda(T)} \\
& \times \int_{t}^{T} \delta^{-2 \lambda(s) / \lambda(T)}\left(\ln \left(\frac{\lambda(T)}{\delta}\right)\right)^{2(\lambda(T)-\lambda(s)) / \lambda(T)} \sum_{k=1}^{\infty}\left|\left(f_{k}\left(u_{\delta}\right)(s)-f_{k}(u)(s)\right)\right|^{2} d s .
\end{aligned}
$$

Hence, we get the following estimates

$$
\begin{aligned}
\frac{3 \pi}{2} \lambda_{1}^{2} L_{1}(t) \leq & 3 \lambda_{1}^{2} \delta^{2 \lambda(t) / \lambda(T)}\left(\ln \left(\frac{\lambda(T)}{\delta}\right)\right)^{2(\lambda(t)-\lambda(T)) / \lambda(T)}\left\|u_{x x}(\cdot, 0)\right\|^{2}, \\
\frac{3 \pi}{2} \lambda_{1}^{2} L_{2}(t) \leq & \frac{3 \pi}{2} T \lambda_{1}^{2} \delta^{2 \lambda(t) / \lambda(T)}\left(\ln \left(\frac{\lambda(T)}{\delta}\right)\right)^{2(\lambda(t)-\lambda(T)) / \lambda(T)} \int_{0}^{T} \sum_{k=1}^{\infty} k^{4} e^{2 k^{2} \lambda(s)} f_{k}^{2}(u)(s) d s, \\
\frac{3 \pi}{2} \lambda_{1}^{2} L_{3}(t) \leq & 3 L^{2} \lambda_{1}^{2} T \delta^{2 \lambda(t) / \lambda(T)}\left(\ln \left(\frac{\lambda(T)}{\delta}\right)\right)^{2(\lambda(t)-\lambda(T)) / \lambda(T)} \\
& \times \int_{t}^{T} \delta^{-2 \lambda(s) / \lambda(T)}\left(\ln \left(\frac{\lambda(T)}{\delta}\right)\right)^{2(\lambda(T)-\lambda(s)) / \lambda(T)}\left\|u(\cdot, s)-u_{\delta}(\cdot, s)\right\|^{2} d s .
\end{aligned}
$$


International Journal of Mathematics and Mathematical Sciences

From the estimate (2.39), we get

$$
\begin{aligned}
\| u(\cdot, t)- & u_{\delta}(\cdot, t) \|^{2} \\
\leq & 3 \lambda_{1}^{2} \delta^{2 \lambda(t) / \lambda(T)}\left(\ln \left(\frac{\lambda(T)}{\delta}\right)\right)^{2(\lambda(t)-\lambda(T)) / \lambda(T)} \\
& \times\left(\left\|u_{x x}(\cdot, 0)\right\|^{2}+\frac{\pi}{2} T \int_{0}^{T} \sum_{k=1}^{\infty} k^{4} e^{2 k^{2} \lambda(s)} f_{k}^{2}(u)(s) d s\right) \\
& +3 L^{2} T \lambda_{1}^{2} \delta^{2 \lambda(t) / \lambda(T)}\left(\ln \left(\frac{\lambda(T)}{\delta}\right)\right)^{2(\lambda(t)-\lambda(T)) / \lambda(T)} \\
& \times \int_{t}^{T} \delta^{-2 \lambda(s) / \lambda(T)}\left(\ln \left(\frac{\lambda(T)}{\delta}\right)\right)^{(2 \lambda(T)-2 \lambda(s)) / \lambda(T)}\left\|u(\cdot, s)-u^{\delta}(\cdot, s)\right\|^{2} d s .
\end{aligned}
$$

Hence, we have

$$
\begin{aligned}
\delta^{-2 \lambda(t) / \lambda(T)}\left(\ln \left(\frac{\lambda(T)}{\delta}\right)\right)^{2(\lambda(T)-\lambda(t)) / \lambda(T)}\left\|u(\cdot, t)-u_{\delta}(\cdot, t)\right\|^{2} \\
\leq 3 \lambda_{1}^{2}\left(\left\|u_{x x}(\cdot, 0)\right\|^{2}+\frac{\pi}{2} T \int_{0}^{T} \sum_{k=1}^{\infty} k^{4} e^{2 k^{2} \lambda(s)} f_{k}^{2}(u)(s) d s\right) \\
\quad+3 L^{2} T \lambda_{1}^{2} \int_{t}^{T} \delta^{-2 \lambda(s) / \lambda(T)}\left(\ln \left(\frac{\lambda(T)}{\delta}\right)\right)^{(2 \lambda(T)-2 \lambda(s)) / \lambda(T)}\left\|u(\cdot, s)-u^{\delta}(\cdot, s)\right\|^{2} d s .
\end{aligned}
$$

Applying Gronwall's inequality, we obtain

$$
\begin{aligned}
& \delta^{-2 \lambda(t) / \lambda(T)}\left(\ln \left(\frac{\lambda(T)}{\delta}\right)\right)^{(2 \lambda(T)-2 \lambda(t)) / \lambda(T)}\left\|u(\cdot, t)-u_{\delta}(\cdot, t)\right\|^{2} \\
& \leq 3 \lambda_{1}^{2} e^{3 L^{2} T \lambda_{1}^{2}(T-t)}\left(\left\|u_{x x}(\cdot, 0)\right\|^{2}+\frac{\pi}{2} T \int_{0}^{T} \sum_{k=1}^{\infty} k^{4} e^{2 k^{2} \lambda(s)} f_{k}^{2}(u)(s) d s\right) .
\end{aligned}
$$

Hence, we get

$$
\begin{aligned}
& \delta^{-2 \lambda(t) / \lambda(T)}\left(\ln \left(\frac{\lambda(T)}{\delta}\right)\right)^{(2 \lambda(T)-2 \lambda(t)) / \lambda(T)}\left\|u(\cdot, t)-u_{\delta}(\cdot, t)\right\|^{2} \\
& \leq 3 \lambda_{1}^{2} e^{3 L^{2} T \lambda_{1}^{2}(T-t)}\left(\left\|u_{x x}(\cdot, 0)\right\|^{2}+\frac{\pi}{2} T \int_{0}^{T} \sum_{k=1}^{\infty} k^{4} e^{2 k^{2} \lambda(s)}\left(f_{k}(u)(s)\right)^{2} d s\right)
\end{aligned}
$$




$$
\begin{aligned}
& =3 \lambda_{1}^{2} e^{3 L^{2} T \lambda_{1}^{2}(T-t)}\left(\left\|u_{x x}(\cdot, 0)\right\|^{2}+\frac{2}{\pi} T \int_{0}^{T} \sum_{k=1}^{\infty} k^{4} e^{2 k^{2} \lambda(s)}\left(\int_{0}^{\pi} f(x, s, u(x, s)) \sin (k x) d x\right)^{2} d s\right) \\
& \leq 3 \lambda_{1}^{2} e^{3 L^{2} T \lambda_{1}^{2}(T-t)}\left(\left\|u_{x x}(\cdot, 0)\right\|^{2}+2 T \int_{0}^{T} \sum_{k=1}^{\infty} k^{4} e^{2 k^{2} \lambda(s)}\left(\int_{0}^{\pi}|f(x, s, u(x, s))|^{2} d x\right) d s\right) .
\end{aligned}
$$

From (1.1), we have

$$
\begin{aligned}
\delta^{-2 \lambda(t) / \lambda(T)}\left(\ln \left(\frac{\lambda(T)}{\delta}\right)\right)^{(2 \lambda(T)-2 \lambda(t)) / \lambda(T)}\left\|u(\cdot, t)-u_{\delta}(\cdot, t)\right\|^{2} \\
\leq 3 \lambda_{1}^{2} e^{3 L^{2} T \lambda_{1}^{2}(T-t)}\left(\left\|u_{x x}(\cdot, 0)\right\|^{2}+2 T \int_{0}^{T} \sum_{k=1}^{\infty} k^{4} e^{2 k^{2} \lambda(s)}\left(\int_{0}^{\pi}|f(x, s, u(x, s))|^{2} d x\right) d s\right) \\
\leq 3 \lambda_{1}^{2} e^{3 L^{2} T \lambda_{1}^{2}(T-t)} \\
\quad \times\left(\left\|u_{x x}(\cdot, 0)\right\|^{2}+2 T \int_{0}^{T} \int_{0}^{\pi} \sum_{k=1}^{\infty}\left|k^{2} e^{k^{2} \lambda(s)}\left(u_{s}(x, s)-a(s) u_{x x}(x, s)\right)\right|^{2} d x d s\right) \\
=Q(t)+M(t),
\end{aligned}
$$

where

$$
\begin{gathered}
Q(t)=3 \lambda_{1}^{2} e^{3 L^{2} T \lambda_{1}^{2}(T-t)}\left\|u_{x x}(\cdot, 0)\right\|^{2} \\
M(t)=6 T \lambda_{1}^{2} e^{3 L^{2} T \lambda_{1}^{2}(T-t)} \int_{0}^{T} \int_{0}^{\pi} \sum_{k=1}^{\infty}\left|k^{2} e^{k^{2} \lambda(s)}\left(u_{s}(x, s)-a(s) u_{x x}(x, s)\right)\right|^{2} d x d s .
\end{gathered}
$$

Therefore, we get the estimate

$$
\left\|u(\cdot, t)-u_{\delta}(\cdot, t)\right\| \leq \sqrt{Q(t)+M(t)} \delta^{\lambda(t) / \lambda(T)}\left(\ln \left(\frac{\lambda(T)}{\delta}\right)\right)^{(\lambda(t)-\lambda(T)) / \lambda(T)} .
$$

Let $v_{\delta}$ be the solution of (1.12) corresponding to the perturbed data $g_{\delta}$, and let $u_{\delta}$ be the solution of (1.12) corresponding to the exact data g. From Theorem 2.3 and (2.46), we can get

$$
\begin{aligned}
\left\|v_{\delta}(\cdot, t)-u(\cdot, t)\right\| & \leq\left\|v_{\delta}(\cdot, t)-u_{\delta}(\cdot, t)\right\|+\left\|u_{\delta}(\cdot, t)-u(\cdot, t)\right\| \\
& \leq \sqrt{2} \lambda_{1} e^{L^{2} \lambda_{1}^{2} T(T-t)} \delta^{\lambda(t) / \lambda(T)}\left(\ln \left(\frac{\lambda(T)}{\delta}\right)\right)^{(\lambda(t)-\lambda(T)) / \lambda(T)}
\end{aligned}
$$


International Journal of Mathematics and Mathematical Sciences

$$
\begin{aligned}
& +\sqrt{Q(t)+M(t)} \delta^{\lambda(t) / \lambda(T)}\left(\ln \left(\frac{\lambda(T)}{\delta}\right)\right)^{(\lambda(t)-\lambda(T)) / \lambda(T)} \\
= & C(t) \delta^{\lambda(t) / \lambda(T)}\left(\ln \left(\frac{\lambda(T)}{\delta}\right)\right)^{(\lambda(t)-\lambda(T)) / \lambda(T)},
\end{aligned}
$$

where $C(t)=\sqrt{2} \lambda_{1} e^{L^{2} \lambda_{1}^{2} T(T-t)}+\sqrt{Q(t)+M(t)}$.

This completes the proof of Theorem 2.4.

\section{Numerical Experiment}

Consider the nonlinear parabolic equation with time-dependent coefficient:

$$
\begin{aligned}
u_{t}(x, t)-a(t) u_{x x}(x, t) & =f(x, t, u(x, t)), \quad(x, t) \in[0, \pi] \times(0,1], \\
u(0, t) & =u(\pi, t)=0, \quad t \in[0,1], \\
u(x, T) & =g(x), \quad x \in[0, \pi]
\end{aligned}
$$

where

$$
\begin{aligned}
a(t) & =(2 t+1), \\
f(x, t, u(x, t)) & =u+4 e^{t} \sin (x) \cos (x)(2 t+1), \\
g(x) & =e \sin (x) \cos (x) .
\end{aligned}
$$

The exact solution of the equation is

$$
u(x, t)=e^{t} \sin (x) \cos (x) .
$$

Letting $t=0$, from (3.3), we have

$$
u(x, 0)=\sin (x) \cos (x)
$$

Consider the measured data

$$
g_{\delta}(x)=\left(1+\frac{\delta}{\|g\|}\right) g(x)=\left(1+\frac{\delta}{1.7034}\right) g(x) \text {. }
$$

Then we have

$$
\left\|g_{\delta}-g\right\|=\delta
$$


Table 1

\begin{tabular}{lc}
\hline$\delta$ & $\left\|v_{\delta_{i}}(n)(\cdot, 0)-u(\cdot, 0)\right\|_{2}$ \\
\hline$\delta_{1}=10^{-1}$ & $3.763414 e-001$ \\
$\delta_{2}=10^{-2}$ & $3.735245 e-001$ \\
$\delta_{3}=10^{-3}$ & $3.475127 e-001$ \\
$\delta_{4}=10^{-4}$ & $2.048545 e-001$ \\
$\delta_{5}=10^{-5}$ & $4.012725 e-002$ \\
$\delta_{6}=10^{-10}$ & $4.491189 e-007$ \\
$\delta_{7}=10^{-20}$ & $2.638466 e-013$ \\
$\delta_{8}=10^{-50}$ & $2.179771 e-013$ \\
\hline
\end{tabular}

From (2.31) and (3.5), we have the regularized solution for the case $t=0$ in the form of iteration

$$
v_{\delta}(n)(\omega, 0)=\sum_{k=1}^{\infty} v_{\delta, k}(n)(x, 0) \sin (k x)
$$

where

$$
\begin{gathered}
v_{\delta, k}(n)(x, 0)=\frac{1}{\alpha_{k}(\delta)+\exp \left\{-2 k^{2}\right\}} g_{\delta, k}-\int_{t}^{1} \frac{\exp \left\{k^{2}\left(s^{2}+s-2\right)\right\}}{\alpha_{k}(\delta)+\exp \left\{-2 k^{2}\right\}} f_{k}\left(v_{\delta}(n-1)\right)(s) d s, \\
g_{\delta, k}=\frac{2}{\pi} \int_{0}^{\pi} g_{\delta}(x) \sin (k x) d x, \\
v_{\delta}(1)(x, 0)=(1+\delta) \sin (x) \cos (x), \\
\alpha_{k}(\delta)=\delta k^{2} .
\end{gathered}
$$

We consider $\delta_{1}=10^{-1}, \delta_{2}=10^{-2}, \delta_{3}=10^{-3}, \delta_{4}=10^{-4}, \delta_{5}=10^{-5}, \delta_{6}=10^{-10}, \delta_{7}=10^{-20}$, $\delta_{8}=10^{-50}$, and $n=10$. Now, we get Table 1 for the case $t=0$.

We have in Figure 1 the graphs of the regularized solution $v_{\delta_{i}}(n)(\cdot, t), i=1,2,3$ and $n=10$. $n=10$.

We have in Figure 2 the graphs of the regularized solution $v_{\delta_{i}}(n)(\cdot, t), i=4,5,6$ and

We have in Figure 3 the graphs of the exact solution $u(\cdot, t)$ and of the regularized solution $v_{\delta_{i}}(n)(\cdot, t), i=7,8$ and $n=10$.

Now, Figure 4 can represent visually the exact solution and regularized solutions corresponding to $\delta_{i}, i=1, \ldots, 8$ at initially time $t=0$.

Notice that, in Figure 4, the curve number 0 expressing the exact solution is indistinguishable from the curve number $i$ expressing the regularized solution corresponding to $\delta_{i}, i=6,7,8$.

Remark 3.1. From (1.7) and (3.5), we obtain the exact solution corresponding to the measured data $g_{\delta}(x)$ :

$$
w(x, t)=\sum_{k=1}^{\infty} w_{k}(t) \sin (k x)
$$




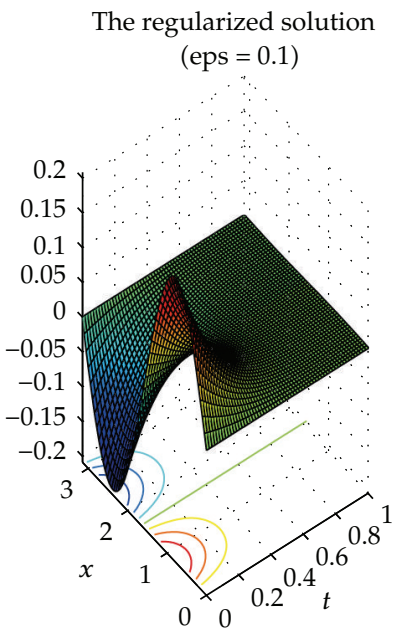

(a)

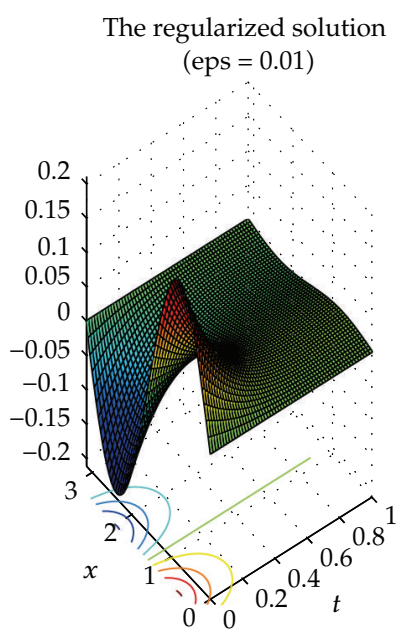

(b)
The regularized solution $($ eps $=0.001)$

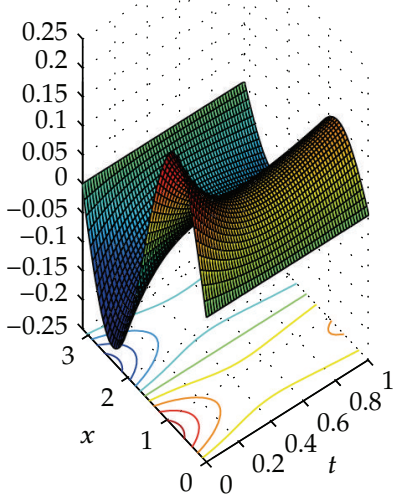

(c)

Figure 1: The regularized solutions corresponding to $\delta_{i}, i=1,2,3$.

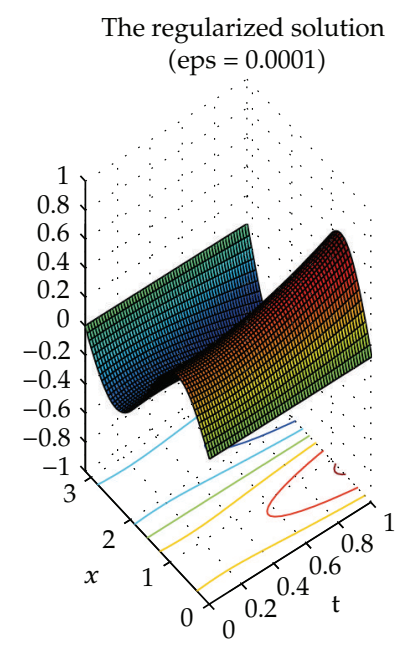

(a)

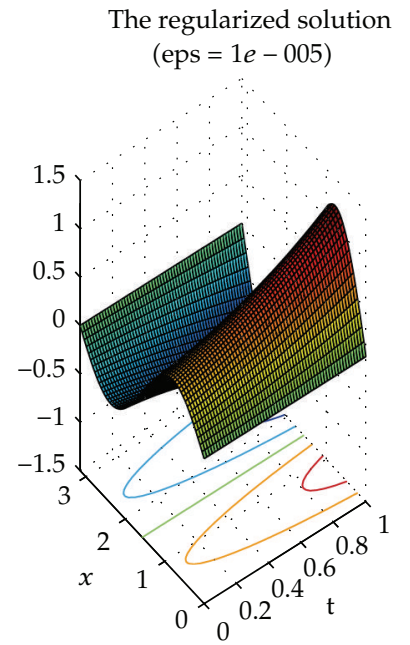

(b)
The regularized solution

$($ eps $=1 e-010)$

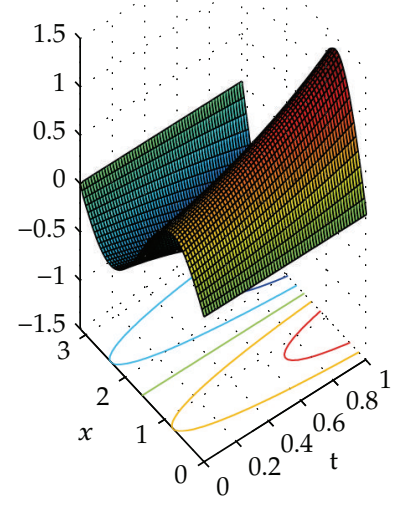

(c)

Figure 2: The regularized solutions corresponding to $\delta_{i}, i=4,5,6$.

where

$$
\begin{aligned}
w_{k}(t) & =e^{k^{2}(\lambda(T)-\lambda(t))} g_{\delta, k}-\int_{t}^{T} e^{k^{2}(\lambda(s)-\lambda(t))} f_{k}(w)(s) d s, \\
f_{k}(w)(s) & =\frac{2}{\pi} \int_{0}^{\pi} f(x, s, w(x, s)) \sin (k x) d x, \\
g_{\delta, k} & =\frac{2}{\pi} \int_{0}^{\pi} g_{\delta}(x) \sin (k x) d x .
\end{aligned}
$$




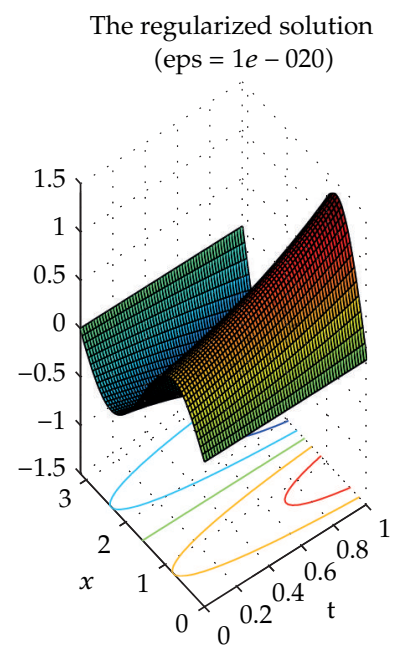

(a)

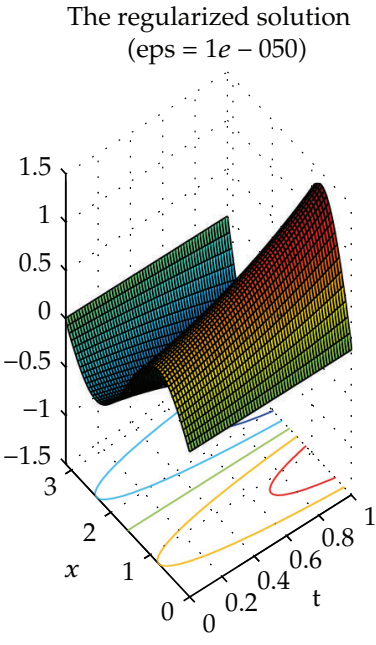

(b)

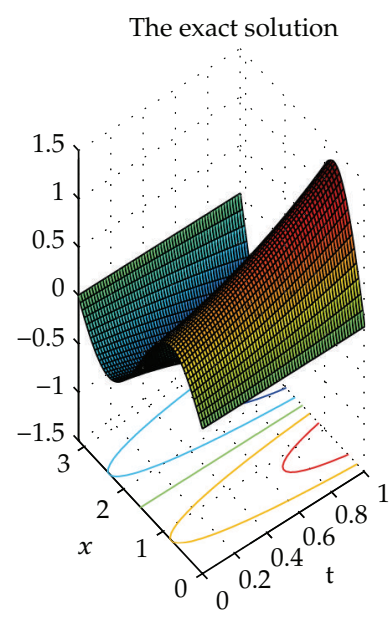

(c)

Figure 3: The regularized solutions corresponding $\delta_{i}, i=7,8$ and the exact solution.

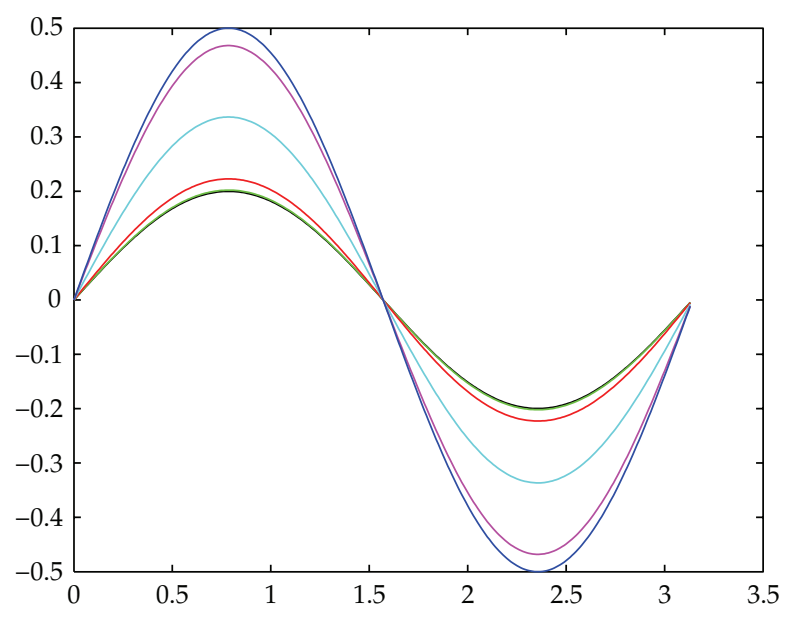

Figure 4: The exact solution and the regularized solutions corresponding to $\delta_{i}, i=1, \ldots, 8$ at initial time $t=0$.

Now, we cannot calculate the formula (3.9) exactly (we need to find $f_{k}(w)$ (s) while we have not known $w$ yet). From Theorem 2.2, we use the iteration for (3.9) at initial time $t=0$ as follows:

$$
w(n)(\omega, 0)=\sum_{k=1}^{\infty} w_{k}(n)(x, 0) \sin (k x)
$$


Table 2

\begin{tabular}{lc}
\hline$\delta$ & $\left\|w_{\mathcal{S}_{i}}(5)(\cdot, 0)-u(\cdot, 0)\right\|_{2}$ \\
\hline$\delta_{1}=10^{-1}$ & $1.1856 e+031$ \\
$\delta_{2}=10^{-2}$ & $1.0 e+031$ \\
$\delta_{3}=10^{-3}$ & $1.0 e+031$ \\
$\delta_{4}=10^{-4}$ & $1.0 e+031$ \\
$\delta_{5}=10^{-5}$ & $1.0 e+031$ \\
$\delta_{6}=10^{-10}$ & $1.0 e+031$ \\
$\delta_{7}=10^{-20}$ & $1.0 e+031$ \\
$\delta_{8}=10^{-50}$ & $1.0 e+031$ \\
\hline
\end{tabular}

where

$$
\begin{gathered}
w_{k}(n)(x, 0)=\exp \left\{2 k^{2}\right\} g_{\delta, k}-\int_{0}^{1} \exp \left\{k^{2}\left(s^{2}+s\right)\right\} f_{k}(w(n-1))(s) d s, \\
g_{\delta, k}=\frac{2}{\pi} \int_{0}^{\pi} g_{\delta}(x) \sin (k x) d x \\
w(1)(x, 0)=(1+\delta) \sin (x) \cos (x) .
\end{gathered}
$$

Then we get the error in the Table 2 .

We can see that the error $\left\|w_{\mathcal{S}_{i}}(5)(\cdot, 0)-u(\cdot, 0)\right\|_{2}$ is very large. Therefore, the problem is ill-posed and a regularization is necessary.

\section{Acknowledgment}

All authors were supported by the National Foundation for Science and Technology Development (NAFOSTED).

\section{References}

[1] Z. Qian, C.-L. Fu, and R. Shi, "A modified method for a backward heat conduction problem," Applied Mathematics and Computation, vol. 185, no. 1, pp. 564-573, 2007.

[2] R. E. Ewing, "The approximation of certain parabolic equations backward in time by Sobolev equations," SIAM Journal on Mathematical Analysis, vol. 6, pp. 283-294, 1975.

[3] X.-L. Feng, Z. Qian, and C.-L. Fu, "Numerical approximation of solution of nonhomogeneous backward heat conduction problem in bounded region," Mathematics and Computers in Simulation, vol. 79, no. 2, pp. 177-188, 2008.

[4] C.-L. Fu, X.-T. Xiong, and Z. Qian, "Fourier regularization for a backward heat equation," Journal of Mathematical Analysis and Applications, vol. 331, no. 1, pp. 472-480, 2007.

[5] D. N. Hào, "A mollification method for ill-posed problems," Numerische Mathematik, vol. 68, no. 4, pp. 469-506, 1994.

[6] A. Hasanov and J. L. Mueller, "A numerical method for backward parabolic problems with nonselfadjoint elliptic operators," Applied Numerical Mathematics, vol. 37, no. 1-2, pp. 55-78, 2001.

[7] D. D. Trong, P. H. Quan, and N. H. Tuan, "A quasi-boundary value method for regularizing nonlinear ill-posed problems," Electronic Journal of Differential Equations, vol. 2009, no. 109, pp. 1-16, 2009.

[8] D. T. Dang and H. T. Nguyen, "Regularization and error estimates for nonhomogeneous backward heat problems," Electronic Journal of Differential Equations, vol. 2006, no. 4, pp. 1-10, 2006. 
[9] I. V. Mel'nikova and A. I. Filinkov, The Cauchy Problem. Three Approaches, vol. 120 of Monograph and Surveys in Pure and Applied Mathematics, Chapman \& Hall, London, UK, 2001.

[10] D. D. Trong and N. H. Tuan, "A nonhomogeneous backward heat problem: regularization and error estimates," Electronic Journal of Differential Equations, vol. 2008, no. 33, pp. 1-14, 2008.

[11] D. D. Trong, P. H. Quan, T. V. Khanh, and N. H. Tuan, "A nonlinear case of the 1-D backward heat problem: regularization and error estimate," Zeitschrift für Analysis und ihre Anwendungen, vol. 26, no. 2, pp. 231-245, 2007.

[12] P. H. Quan, D. D. Trong, L. M. Triet, and N. H. Tuan, “A modified quasi-boundary value method for regularizing of a backward problem with time-dependent coefficient," Inverse Problems in Science and Engineering, vol. 19, no. 3, pp. 409-423, 2011.

[13] M. Lees and M. H. Protter, "Unique continuation for parabolic differential equations and inequalities," Duke Mathematical Journal, vol. 28, pp. 369-382, 1961. 


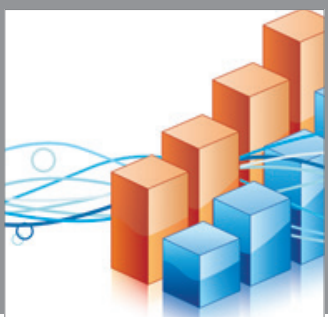

Advances in

Operations Research

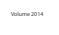

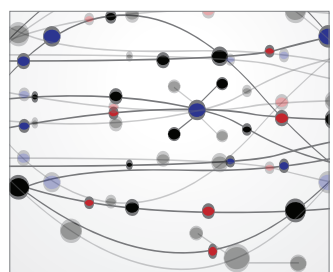

\section{The Scientific} World Journal
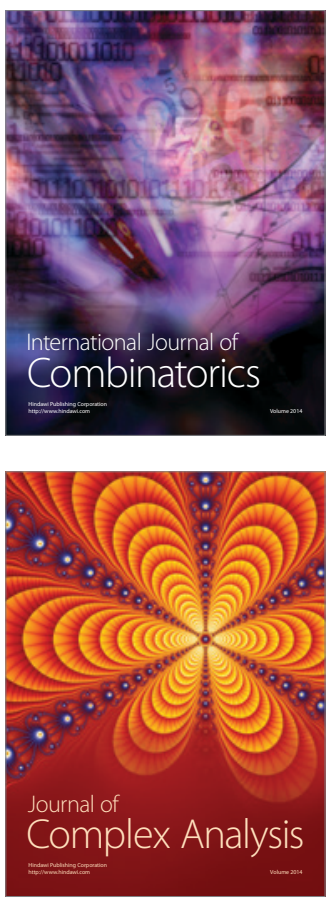

International Journal of

Mathematics and

Mathematical

Sciences
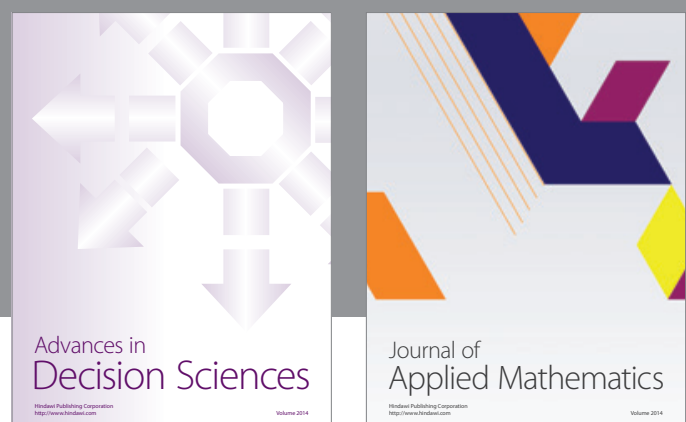

Journal of

Applied Mathematics
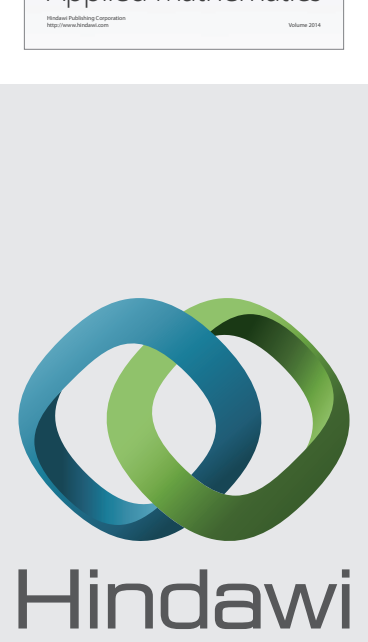

Submit your manuscripts at http://www.hindawi.com
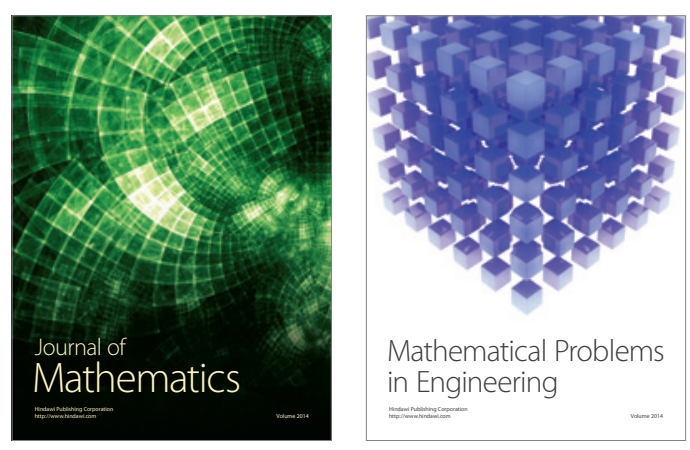

Mathematical Problems in Engineering
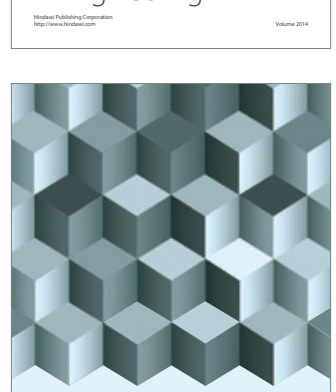

Journal of

Function Spaces
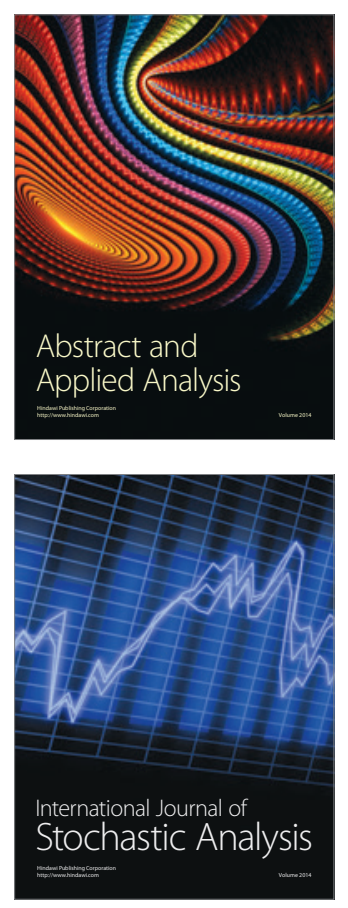

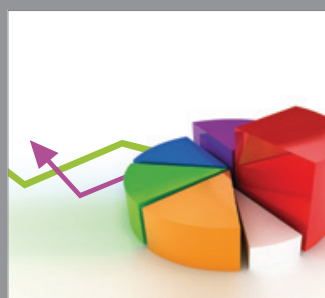

ournal of

Probability and Statistics

Promensencen
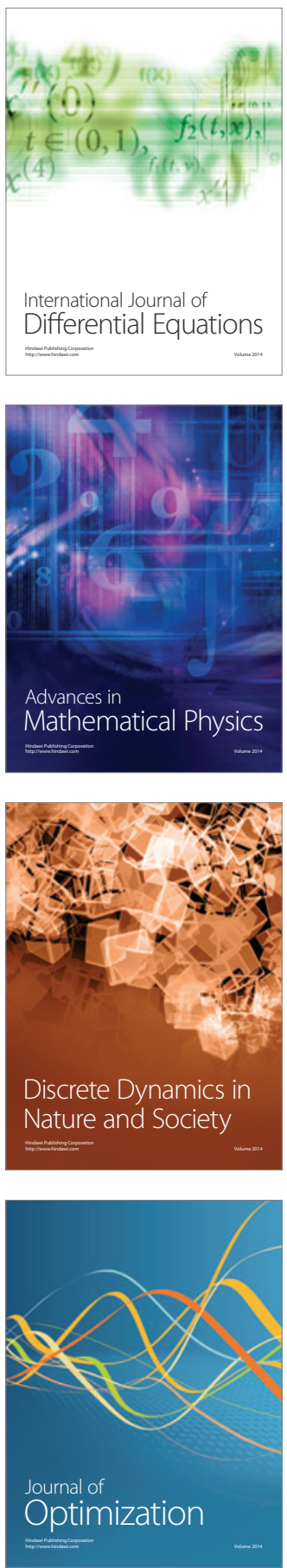